\title{
A Technological Approach for Evaluating the Sustainability Performance of Housing Developments
}

\author{
Ioanna Dionysiou, Philippos Pouyioutas, Harald Gjermundrod, Chrystalla Miltiadou, \\ Theodora Ioannou \\ Department of Computer Science, University of Nicosia, Nicosia, Cyprus
}

\begin{abstract}
This paper discusses a software application that will assist those involved in any of the stages of the housing development lifespan to make informed decisions based on the sustainability assessment of the entire development. This will lead to harmonization of the design of housing compounds with the environmental design and sustainability parameters and to the promotion of sustainable development practices.
\end{abstract}

\section{Introduction}

In order to ensure the life quality of future generations, according to the sustainability paradigm, it is essential to secure housing development in such a way that the continuity of natural resources production, quality and existence will be retained. This can only be accomplished through sustainable housing and industrial development. The incorporation of sustainability principles in the design of neighborhoods, communities and cities is crucial and must be applied during all phases in the lifespan of a housing development. This will lead to harmonization of design of housing developments with the environmental design and sustainability parameters and promotion of sustainable development practices.

The focal point of the paper is the technological advancement in the area of sustainable development by presenting an innovative user-friendly application that assesses the sustainability of the built environment. The application directly contributes towards a controlled town planning by the planning of housing developments within the context of environmental design and sustainable development parameters, and thus yielding to the environmental upgrading of towns. Existing urban settings could also be upgraded, whereas the design and planning of new developments, regardless of the size, could take into consideration special environmental and social conditions. Thus, those involved into any stage of the housing development lifespan could make informed decisions based on the generated sustainability assessment.

The application will clearly indicate the sustainability variables that could be further improved in order to accomplish higher sustainability performance by fine-tuning the sustainability parameters and their associated weights. Using a mathematical model, which constitutes an innovation in the section of sustainable housing development, the application exploits it and turns it into a practical application.

The remaining of the paper is organized as follows: Section 2 discusses the importance of sustainable housing development. Section 3 discusses the system requirements. Section 4 presents the design logistics of the software application that evaluates a housing development based on environmental design principles, followed by the implementation details of this application in Section 5. Section 6 presents the future direction for the application. The concluding remarks are given in Section 7.

\section{Need for Sustainable Housing Development}

Over the last few decades, governments, researchers, scientists and environmental organizations are being alarmed of the hazardous consequences the planet is facing today due to the careless, anthropocentric and uncontrolled environmental attitudes towards nature. Humans have always caused severe damages to the environment and its resources by excessively utilizing and exploiting them, while not being aware of the negative impacts that could have possibly be done and not taking into consideration the importance for implementing measures towards exploiting renewable resources or finding alternatives that would be harmless. Extraction of raw materials from the environment, air emissions, waste disposal and deforestation are some of the most common causes that civil growth and development have had on the environment we live in.

Due to the fact that limiting growth was never a choice, a new innovative concept was introduced by the Brundtland Commission (formerly known as the World Commission on Environment and Development) that would allow friendly environmental development and policies. In their 1987 report "Our Common Future" sustainable development was defined as "the process to meet the needs of the present generation without compromising the needs of future generations" [1]. It was suggested that sustainable development has the potential of a new alternative way that allows the maximum possible use of environmental resources, but at the same time sustains the availability of the same resources in the same quantity for the upcoming generations. Major parts of sustainable 
development is the inevitable technological improvement that takes into consideration the environmental values as well as the economic development, to assist countries to achieve their goals and apply environmental policies that would assist them to improve their quality of life.

The Environmental Sustainability Index (ESI) [2] and its successor Environmental Performance Index (EPI) [3] are methods of quantifying and numerically benchmarking the environmental performance of a country's policies. In a sense, they track a number of pre-defined indicators, which are then aggregated to obtain the overall performance for a country. Both indexes were developed by Yale University and Columbia University in collaboration with the World Economic Forum and the Joint Research Centre of the European Commission.

The Environmental Sustainability Index method has as a primary goal to indicate whether or not countries will be able to shield the environment for the upcoming decades. In order to do that, ESI tracks 21 environmental sustainability elements (indices) that refer to natural resource endowments, past and present pollution levels, environmental management efforts and the rate at which a society can improve its environmental performance. These indices fall into five categories: Environmental Systems, reducing environmental stresses, reducing human vulnerability, social and institutional capacity, and global stewardship. A total of 76 variables are used to form a complete environmental policy model that would allow the sustainability factor to be measured by calculating the score of each country. High scores indicate high performance, whereas a low score indicates low performance.

The need for better and more precise performance evaluation method led to the development of the Environmental Performance Index (EPI) that not only could be used more easily by policy makers and scientists, but would be more accurate as well. EPI aimed to fill the gaps, such as missing data gaps or out-of-date data, that prevented policy makers from spotting out emerging problems, evaluating policy options or measuring the effectiveness of good environmental planning. The two main environmental objectives that EPI focuses on are reducing environmental stresses on human health and promoting the ecosystem vitality and natural resource management.

There have been released two EPI reports so far, one in 2008 [4] and another one in 2010 [5] that rank countries based on their sustainability performance. The EPI 2008 report used 25 indicators that would offer an overview of a country's environmental challenges as it seeks to meet the needs of governments to track on-the-ground environmental results. It supports a method to assess the effectiveness of environmental policies against relevant performance goals. Therefore, EPI 2008 would use a method called "proximity-to-target" to obtain a value for the indicators that would be calculated by finding the difference between a country's policy targets and its current results. However, even though it seemed to be an efficient approach, due to the lack of reliable measures and data, only 149 countries out of 238 could be evaluated with EPI 2008. EPI 2010 is not as restricted as EPI 2008 when it comes to missing data. EPI 2010 can track performance changes over time based on a "time-series-data", thus it allows comparisons to be made to see which countries are doing better over time and which ones are falling behind (measuring progress).

Since housing developments is an integral part of a country, upgrading the environmental sustainability performance of the first can substantially affect the overall sustainability performance of the latter. The application of Environmental Design principles [6] (i.e. smaller buildings creation, use of recyclable and renewable materials, use of low embodied energy materials, use of harvest timber, water collection systems implementation, use of low maintenance materials, buildings' recycling, reduction of ozone's chemical destruction, maximizing natural environment sustenance, energy efficiency accomplishment, implement buildings' orientation, public transport access provision) can substantially contribute in sustainable housing development [7].

Considering that housing development sustainability is a broad and abstract term, the methodology developed in [7] could be used as a tool to design sustainable housing developments and evaluate existing ones. The methodology is based on the Environmental Design principles [6], ESI [3], social attitudes and perceptions that people expressed regarding the above $[8,9,10,11]$, and sustainability principles [12]. The methodology defines variables that must be taken into consideration during the planning and design of housing developments. These variables are categorized into sustainability indexes that fall into the categories of priorities set by the Habitat Agenda [12]. The methodology concludes to a mathematical model for the arithmetic evaluation of the sustainability performance of a housing development. To the best of our knowledge, the only software application that implements this methodology is the one presented in this paper.

\section{Software Application Requirements}

As it was mentioned before, the application must have an intuitive interface to accommodate users with no particular scientific and technical knowledge regarding environmental and sustainable development. Users (independent engineers, land planners, town planners, land developing companies, competent authorities and other involved parties in the sector of housing and town development and 
upgrading) will familiarize themselves with environmental and sustainability principles and variables, which will result in increasing their awareness and knowledge levels regarding sustainable development and as a consequence, enhancing their design skills to create more environmental-friendly buildings, neighborhoods, communities and cities.

Details of the mathematical model that the application deploys as well as the system requirements are now presented.

\subsection{Sustainability Housing Development Mathematical Model}

The application combines the existing knowledge and experience in the field of housing sustainability with information technology to provide a new, original, and innovative tool for applied sustainability, by taking into consideration critical variables that can be measured, evaluated and applied in the built environment [7]. Development housing sustainability variables and indexes are determined and formulated, an arithmetic evaluation scale for the sustainability variables is defined, and a mathematic formula for the weighted-based arithmetic estimation of sustainability performance of housing developments is formulated.

In a nutshell, the system will calculate the sustainability performance of housing developments. A particular housing will be evaluated using variables that will be configured by an administrator. Each one of those variables belongs to an index, and each one of the indexes belongs to a category. The mathematical model consists of 5 categories, 24 indexes, and 105 variables. The mathematical expression that assesses the sustainability performance $\mathrm{S}$ of a housing development is shown in (1). V is the actual value of variable $i$, and $f$ is the value of the associated environment influencing factor for the specific variable. $\mathrm{R}$ is the regional importance for variable $\mathrm{i}$. In case of incomplete data, the model utilizes parameters $\mathrm{M}$ and $\mathrm{N}$ (if all data is known, then those parameters are set to 1 ).

$$
S=\sum_{i=1}^{k} M_{i} N_{i} R_{i} V_{i} f_{i} \quad \text { with } \mathrm{k}=105
$$

Details on the mathematical model can be found in [7].

\subsection{System Requirements}

Major inputs to the software are information details about the housing, the default variable values and names, indexes and categories, and also the different evaluation variable weights for the housing that will be evaluated. Major outputs of the system are information on a housing evaluation as well as a list of housings along with their details. Analytically, the system must support the following features:

\section{Interface Requirements}

The system supports both administrator and user functionality. Thus, the administrator-related tasks should appear only for an administrator, whereas user-related tasks would be available to a user.

One of the primary objectives is to design a software tool that with little or no training it would be easy to use, through an assistive and friendly interface that would provide recognition rather than recall. Furthermore, Human Computer Interaction concepts will be applied during the phase of interface design. Fields will be aligned and spaced in a way to give consistency and aesthetics, and at the same time a minimalistic design. In addition, the colors that will be used are warm and friendly colors. As for the main menu, it will be very simple and easy to understand, by providing affordance with its carefully selected icons. All data will be inserted through forms and commands will be issued via buttons, while feedback messages will acknowledge user's interactions.

\section{Functional Requirements}

The system is expected to support the following tasks:

- Management of sustainability variables and indexes, along with their values and weights, for housing developments

- Evaluation of sustainability performance for a housing development based on the mathematical model

- Maintenance of records of all evaluations performed on housing developments, thus enabling the comparison and contrast among housing developments, as well as the monitoring of the sustainability performance of a particular housing development.

\section{Performance Requirements}

The system will need a robust and scalable database management system (DBMS) to maintain the data collected by the users. A current requirement for the system that would host the database could be:

- At least $100 \mathrm{~GB}$ for smooth operation

- Additional 100GB hard drive for back up

- A small business rack server

The system primarily deals with storage and retrieval of small sets of data, and rarely complex transactions. Therefore, performance will not become an issue.

\section{Security Requirements}

The system will support role-based authentication, where users will be authorized to access system 
features based on their role. The authentication credentials are username and password.

\section{Software Application Design}

This section discusses the design choices for the application, with special focus on Data Flow Diagrams and the database design.

\subsection{Data Flow Diagrams}

The system design is done using Data Flow Diagrams (DFDs). A data flow diagram is a graphical representation of the system's processes and activities in general. It shows the functionality of a system in order to visualize and understand the various processes of data, how data flows during the execution of the system, and presents the interaction between the user and the system. Due to space limitations, only a small subset of DFDs could be presented here. The complete set of DFDs can be found in [13].

Fig. 1 illustrates the system functionality for the administrator task that manages the sustainability development variables. First, the administrator must enter his/her credentials, which will be processed according to the authentication scheme. If there is a positive match, the administrator has been authenticated. The next step is to choose the category and index in which the particular variable belongs to. After that, the administrator can add, delete or change the variables. If an addition, deletion or change of information is done, then the data repository that holds all the information about variables will be updated accordingly.

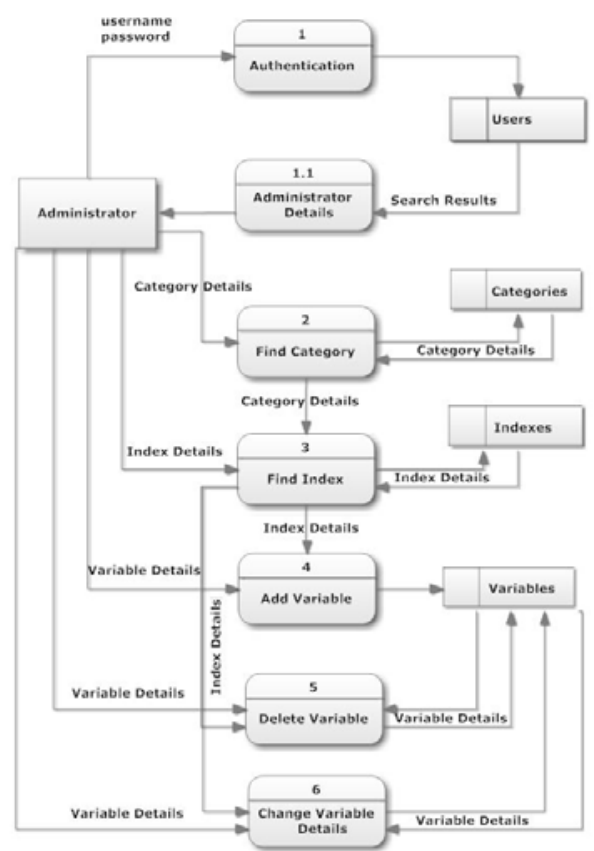

Figure 1. DFD for variable management by administrator

For a normal user, managing Categories, Indexes and Variables is done differently from the one explained above. The data flow diagram in Fig. 2 represents the user's functionalities.

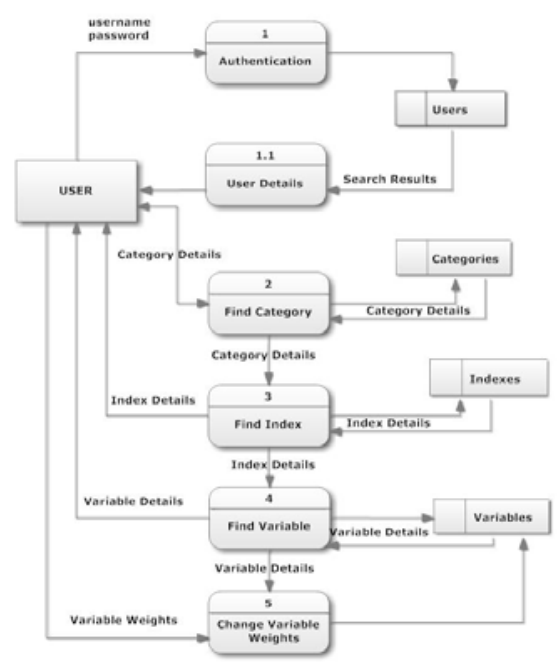

Figure 2. DFD for variable configuration by user

Upon successful authentication, the user can only view categories since $\mathrm{s}$ /he has no privileges for adding or deleting data. User can view all the categories that exist, and choose to find its indexes, which in turn cannot be modified. Similarly, users can find variables that belong to an index, but without adding or deleting any variables; the only thing that can be done is to change the default variable's weights (and value) in the system. 


\subsection{Database Design}

The database design is one of the most crucial and important parts of software development since it can be assumed as the basic structure that the system will be built on. An entity relationship diagram (ERD) is used to capture the database design for the proposed software. An entity relationship diagram is a diagram that represents the relational schema of a database. It consists of four basic parts, which are entities, their attributes, relationships and their cardinalities. Entity can be defined as anything, real or abstract, that users want to track and to be visible by any other object within the database. Entities can have one or more attributes that are descriptions to an entity's characteristics. Entities can interact with one another, thus relationships could be defined between them to describe the connections. In order to define the relationships among entities, there is a need to set their cardinalities that describe how many entities of a specific type correspond to the entities of another type.

Fig. 3 illustrates the ERD for the application that has three entities for Categories, Indexes, and Variables with two relationships between them and four entities for Housing and Evaluation with three relationships amongst them. The system stores several data records that are used to calculate the sustainability factor of different housings.

There are 105 variables in total that define (through an evaluation function) the sustainability factor of housings. Those 105 variables are separated and grouped under one index (each variable belongs to an index that is defined by the administrator). Every index, in turn, belongs to a specific category. As shown in the ERD of Fig. 3, Category table has a primary key Category_ID, which is a unique number for each category that also has a unique name Category_Name that the administrator will specify during system configuration.

One category though, as mentioned before, can have one or more indices. The Index table consists the Index_ID that is the primary key and the name of the index Index_Name. An index can have one or more variables and it is again the task of the administrator to define which variables belong to the specific index. A variable has a variable_ID, which will uniquely identify each variable. The Variable table also stores a Variable Value, and three variable weights Variable_F_Weight, Variable_N_Weight, and Variable_M_Weight as well as the Regional Importance weight Regional_Importance_weight, as these values are going to be used by the mathematical model to calculate the sustainability factor of a housing development.

The user along with the variable value will introduce the above weights into the system whenever a new housing is set up. Housing will have its own ID Housing_ID, its name Housing_Name, the date that is created, the country it belongs to, the city and the region of the city. A description field is used to store any information, details or comments the user may have about the housing. Since Housing consists of different types of structures at different locations and addresses, a new table called Item is responsible to store all the information about a specific structure. The Item table has fields for choosing the type of structure, the exact location or address where the structure is located. In addition, it also saves the coordinates $\mathrm{X}$ and $\mathrm{Y}$ of the master plan for each item, as this could be also stored in the database. A description field allows the user to add any comments or information about an item.

The main purpose of the software is to calculate the sustainability factor for each one of the housings that exist or may be added into the system. Therefore, an Evaluation table should be created to save the results of the evaluation as well as any notes. The Evaluation_Variables table is the one that has all evaluation details. Hence it has fields to store the Evaluation_ID, the Housing_ID, Valiable_ID, Variable_Name and the variable's values and weights (Variable_Value, Variable_F_Weight, Variable_N_Weight, Variable_M_Weight, Regional_Importance_Weight).
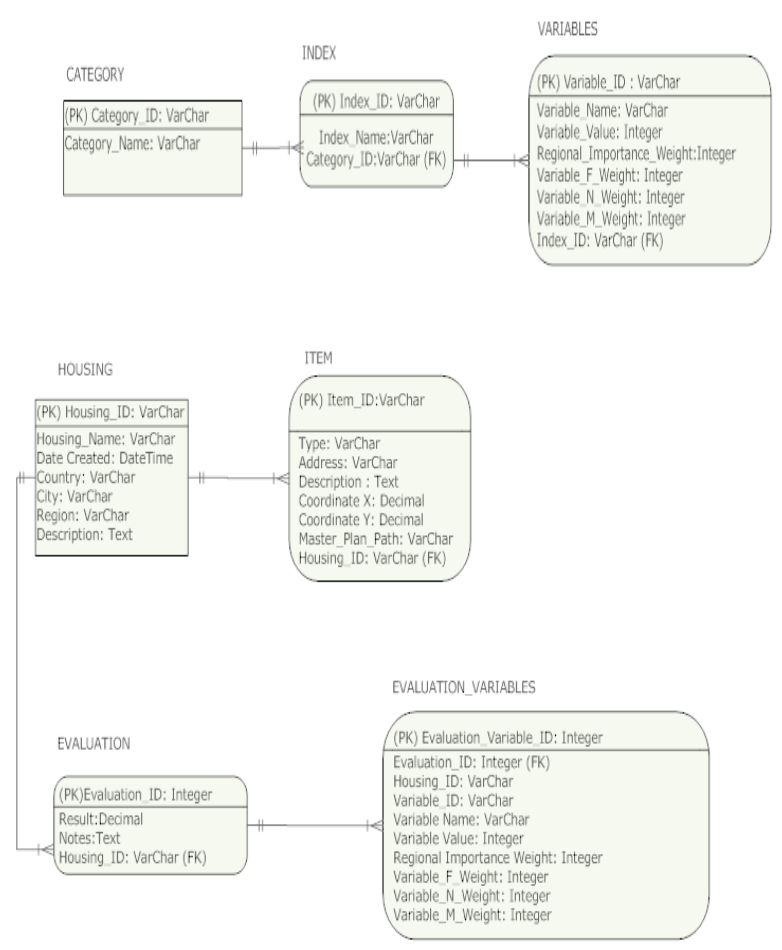

Figure 3. Entity Relationship Diagram for the System

The database relations, after normalization, are the ones below: 


\section{CATEGORY (Category_ID, Category_Name) INDEX (Index_ID, Index_Name, Category_ID) VARIABLES(Variable_ID, Variable_Name, Variable_Value, Regional_Importance_Weight, Variable_F_Value, Variable_N_Value, Variable_M_Value,Index_ID) HOUSING (Housing_ID, Housing_Name, Date_Created, Country, Region, Description) ITEM (Item_ID, Type, Address, Description, Coordinate_X, Coordinate_Y, MasterPlanPath, Housing_ID) \\ EVALUATION (Evaluation_ID, Result, Notes, Housing_ID) \\ EVALUATION_VARIABLES \\ (EvaluationVariables_ID, Evaluation_ID, Housing_ID, Variable_ID, Variable_Name, Variable_Value, Regional_Importance_Weight, Variable_F_Weight, Variable_N_Weight, Variable_M, Weight)}

\section{Software Implementation Details}

The client application was implemented using the Visual Basic 2008 development environment. The Microsoft SQL Server R2 Express was used as the database management system. Before any of the main functionalities of the system can be used, the user must first successfully log in using a valid username and password. In case of incorrect credentials, a message will appear informing the user for the invalid username or password. If, on the other hand, credentials used are valid, the system will check whether the specific person is an administrator or normal user.

A very simple and minimalistic design was used for designing the main menu form. Simplicity but also carefully selected pictures that provide an actual representation of what each choice stands for, easily assist the users in using the software without any prior training.

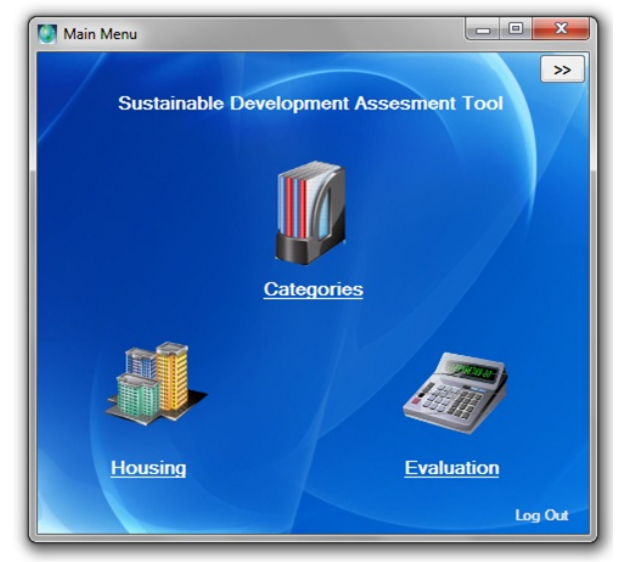

Figure 4. Main menu screen
As shown in Fig. 4, the main form of the application consists of three choices: Category, Housing, and Evaluation. Category, as the name implies, deals with the category operations as specified in the system requirements section. Housing allows tasks related to housing development, like adding new or deleting existing one(s). The Evaluation choice, similarly to the two previous choices, allows the user to do any actions desired for the housing evaluation.

The functionality of the administrator, for some parts of the system, is different than that of a normal user. The Categories functionality is one of those, since the administrator is the only one that can add, update or delete a category. The user can only view the categories without modifying them. Fig. 5 depicts the form that appears on the screen after Categories choice is selected from the main menu. The navigator on the form is used as follows. The left and right arrows are used for moving along the already existing records. The add sign is used to create a new record by filling-in the required information. After filling-in the required textboxes, the save sign icon must be pressed in order to save the changes to the database. The red X sign is for deleting the selected category. In order to prevent any incorrect deletions, the save button must be pressed again to update the database after introducing new changes. According to Fig. 5, there are currently 5 categories stored in the system, as listed in the lower half of the form, namely CAT1, CAT2, CAT3, CAT4, and CAT5. Each category consists of a set of indexes, thus the presence of the Indexes button the upper right corner.

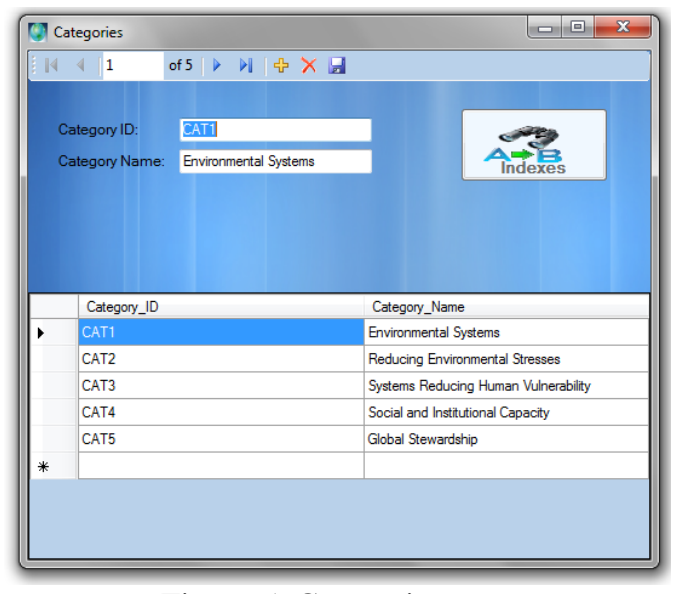

Figure 5. Categories screen

In order to configure the indexes belonging to a category, the particular category is selected and the indexes button is pressed. Suppose CAT4 - Social and Institutional Capacity is selected. Fig. 6 shows the eight indexes that belong to this category, along with their explicit names. In order to specify the variables that populate an index, the Variables button is pressed. 


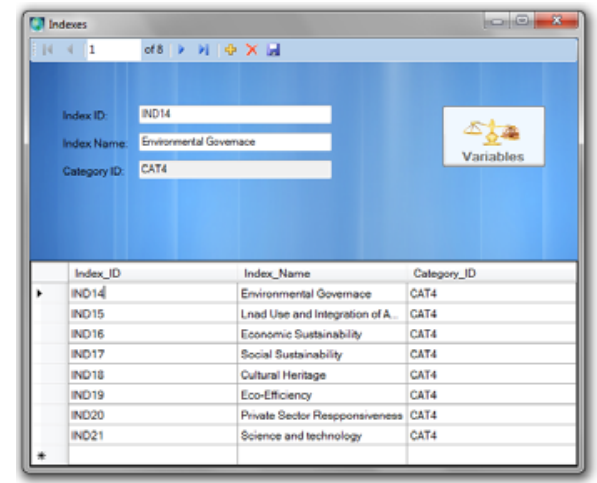

Figure 6. Indexes of category CAT1

Fig. 7 lists the variables for Index IND17. For each variable, there is information regarding its name, value, and its associated weights. For instance, variable VAR66 - level of permeability has default value 1 and all its weights are set to 1 as well.

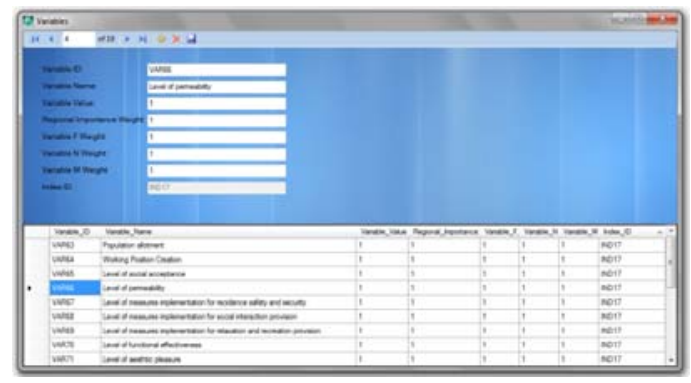

Figure 7. Variables of index IND17

After the administrator configures the categories, indexes, and variables, a user could proceed with evaluating the sustainability performance of a housing development. The first step is to create a new record for the housing development (Fig. 8, Fig. 9), and the second step is to enter values and weights for all variables (Fig. 10). On the upper half of Fig. 8, the information for the Shoreline Hills housing development, located at Limassol, Cyprus is shown.

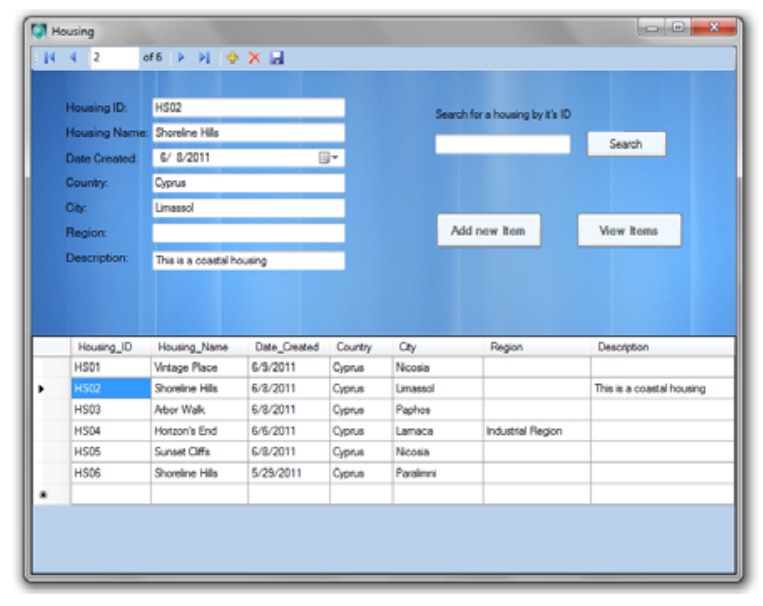

Figure 8. Housing information for shoreline hills
Fig. 9 lists specifically all buildings and green areas that comprise Shoreline Hills: one hotel, two houses, and a park. For each item, the actual coordinates of the structure are entered, along with the master plan for the housing structure, if available. It is important to verify that the new item's ID does not already belong to another housing entry. In such a case, a warning pop up window notifies about the inconsistency. In order to easily identify which housing was selected beforehand, a read-only textbox brings the ID of the housing from the previous form. Also the name of the housing is shown to the right side of the ID. Due to the fact that an item has coordinates to define its location, a link in the upper right corner allows the user to view the item's location using either the coordinates or address on Google Map. Internet explorer starts the Google Map web site with the item's address as the parameter (in the later case).

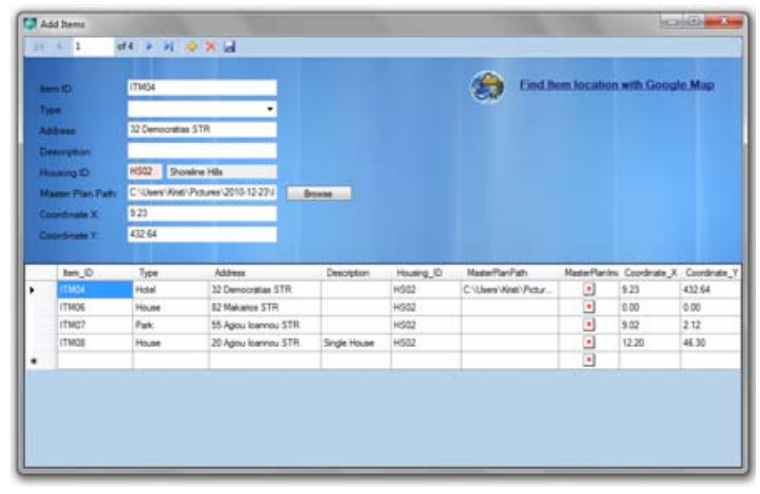

Figure 9. Structures of shoreline hills

Once the housing development is configured, an evaluation could be performed. The Evaluation form (Fig. 10) is activated when the user selects the Evaluation choice from the main menu. Evaluations could be created, viewed, or modified. There is consistency, like in the rest of the forms, with the binding navigator, handling additions, deletions and updates. The add sign is responsible for adding a new evaluation with required fields being the Evaluation ID (to track a number for the evaluation), and the Housing ID (to refer for which Housing development the evaluation is made). Notes is an optional field to be filled, but can be very helpful in the case the user wants to write some comments or observations about the evaluation of a housing. The evaluation result is a required field and it is obtained when the user calculates it. In Fig. 10, there is a list of 6 evaluations: 1 evaluation for HS01 and HS03, and 2 evaluations for HS04 and HS05. 


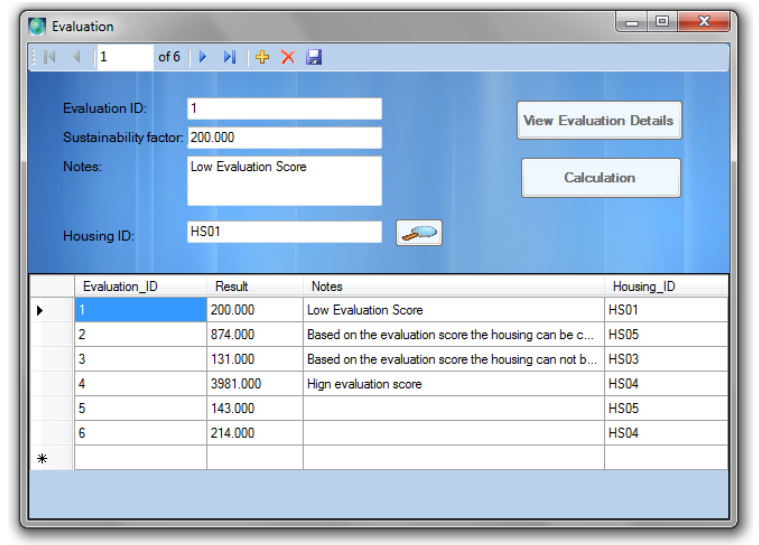

Figure 10. Evaluation screen

The Evaluation form also consists of two important buttons. The first button is the "View Evaluation Details". When the View Evaluation Details button is clicked a new form appears where the user is allowed to view the details for an evaluation. Those details are actually the variables' values and weights used for the calculation of the sustainability factor of a housing entry. Fig. 11 illustrates a snapshot of the values and weights of variables for Housing HSD5. When the "Calculation" button is clicked (Fig. 10), the summation function will be initiated and the sustainability performance score will be displayed. For HS01, the score is 200.

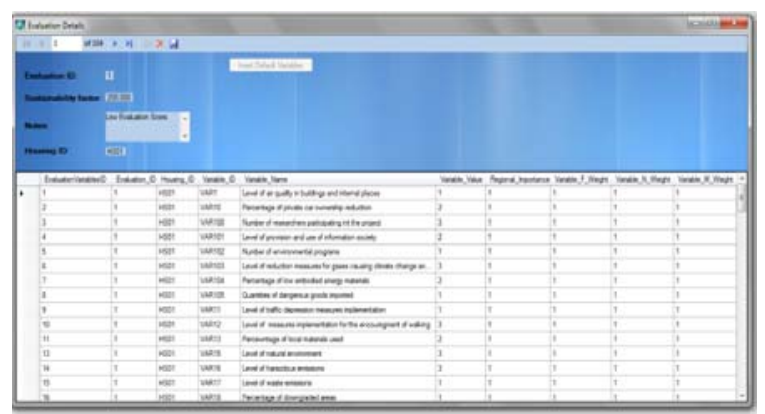

Figure 11. Evaluation details for HS01

\section{Future Directions}

There are a number of improvements that could leverage the existing application's functionality. These include, among others, the following:

- Add more functionality regarding evaluation details: A possible scenario would be to create a scaling system (i.e. 0$10,10-20$, etc.) that would map the evaluated housings to classes (i.e. A, B, C, $\mathrm{D}, \mathrm{E}$ etc. The categorization will be done based on the evaluation score and scale. Thereafter, the class that the housing belongs to will indicate if the housing fulfills the criteria before it is developed. If for example, housing $\mathrm{X}$ would belong to class A, then this housing could be developed whereas if housing $\mathrm{X}$ belonged to class E, it should not be developed. In the case that the housing is an existing one, and the evaluation determines that it belongs to a low class, the system should be able to inform the user that for the specific housing special actions should be taken and some possible actions should also be provided.

- Create crystal reports: Another future plan is to create crystal reports that will allow the user to have a graphical representation of data using charts and diagrams so that it would be easy to get some statistics regarding housings performance.

The system presented in this paper is a standalone application. However, the system could be implemented as a three-tier web application. In this case the application can be accessed from any platform (including smart phones) as long as the user has access to a web browser. The client end will connect to the web application using a standard web browser. The application will have an intuitive and easy-touse interface for the user (tier one). The application logic, i.e. the application functionality, will be provided by the web service that the client application connects to (tier two). It will perform the requests on the behalf of the user. When data is needed or the user provides new data, then it is retrieved or inserted into the back-end database service (tier three). The system will provide for multiple user roles. One role could be assigned to the general public, i.e. anyone who will be able to view the public data stored by the web application. Another role will be authenticated users who will have write access to the information depending on their responsibility, like construction company employees, city planning employees, administrators, etc. Needless to say, multiple users will be able to use the web application simultaneously. The following two activities will lead into a complete implementation of the web application:

- Client Application Development: The client application will be developed using the Microsoft .Net 2010 platform. This platform provides libraries and toolkits for rapid application development. The client application development will be separated into two parts, namely web interface development (tier one) and functionality development (tier two). The .Net platform provides a drag-and-drop web interface development editor with a toolkit of predeveloped GUI elements like buttons, textboxes, menu bar, etc. By using this editor and toolkits the web interface for the proposed web application will get a 
professional look-and-feel and provide an easy-to-use interface that the users are already familiar with. The ASP.NET platform will be used with the C\# programming language to develop the application functionality. ASP.Net provides an object-oriented method for creating and interacting with Web pages. The developed client web application when compiled will be converted into standard HTML. Hence, the client application can be accessed from any platform through an Internet browser like, FireFox, Safari, Opera, Chrome, or Internet Explorer.

- Database Development: The web application will store its information in a back-end database (tier three), which will be accessed by the web service on behalf of the user. Microsoft's SQL Server 2008 R2, which is a DBMS (database management system), will be used as the database service. This DBMS provides seamless integration with the ASP.NET web service.

\section{Conclusions}

Currently in Cyprus, there is little progress towards sustainable housing, primarily due to a number of reasons: (a) there are many requirements regarding the environmental protection and sustainable development (with knowledge of perhaps a few), (b) no sustainable housing development has been planned or constructed in Cyprus, and (c) involved parties (engineers, town planners, Competent Authorities, etc) do not have a satisfactory level of awareness and knowledge of housing sustainable development and environmental design principles. The authors strongly believe that the presented software application would contribute significantly to the inversion of the above, especially nowadays that we are living in a digital era.

The purpose of this application was to apply theoretical concepts of sustainable development to implement a system that would be able to calculate the sustainability performance of a housing development. As far as we know, this is the first software that deploys the mathematical model of [7] to determine the sustainability factor of a housing development. Existing techniques such as the ESI and EPI only allowed the evaluation of a country as a whole, but not separately for each housing development.

\section{References}

[1] World Commission on Environment and Development (WCED), (1987). “Our Common Future”, Oxford: Oxford Univeristy Press, ISBN 0-19-282080-X.
[2] Yale Center for Environmental Law \& Policy and Center for International Earth Science Information Network at Columbia University, (2005). "Environmental Sustainability Index", http://sedac.ciesin.columbia.edu/es/esi/ESI2005_Main_Re port.pdf, Retrieved 2011-05-25.

[3] Yale Center for Environmental Law \& Policy and Center for International Earth Science Information Network at Columbia University, (2010). "Environmental Performance Index”, http://epi.yale.edu/, Retrieved 201105-25.

[4] Yale Center for Environmental Law \& Policy and Center for International Earth Science Information Network at Columbia University, (2008). "2008 Environmental Performance Index Report", http://www.yale.edu/epi/files/2008EPI_Text.pdf, Retrieved 2011-05-25.

[5] Yale Center for Environmental Law \& Policy and Center for International Earth Science Information Network at Columbia University, (2010). "2010 Environmental Performance Index Report", ", http://www.yale.edu/epi/files/2010EPI_Text.pdf, Retrieved 2011-05-25.

[6] Kosmopoulos, P., (2004). Environmental Design, Thessaloniki, University Studio Press.

[7] Ioannou Th., (2011). "Sustainable Housing Development-The Contribution of Environmental Design, Pilot Study on Model Housing”, PhD Thesis, Technical University of Xanthi, Democritus University of Thrace.

[8] Ioannou T. and Kosmopoulos P., (2005). "Environmental Design and Social Reception", 2nd Environmental Conference of Macedonia, Thessaloniki, October 2005.

[9] Ioannou T. and Kosmopoulos P., (2005). "Social Attitudes about Environmental Design and Renewable Energy Sources - Field Study in Cyprus”, International Conference On Passive And Low Energy Cooling For The Built Environment, Santorini, May 2005.

[10] Ioannou T. and Kosmopoulos P., (2005). "Comparative Evaluation of Social Attitudes Regarding Renewable Energy Sources of Specific Groups in Greece and Cyprus", 13th International Symposium On Environmental Pollution And Its Impact On Life In The Mediterranean Region, Thessaloniki, October 2005

[11] Ioannou T. and Kosmopoulos P., (2005). "Social Status Investigation about Energy Conservation”, HELECO '05-5th International Conference for Environmental Technologies, Athens, February 2005.

[12] UN,(1996). Habitat Agenda, http://www.unescap.org/huset/habitat.html, Retrieved 2011-05-25.

[13] Miltiadou, Chrystalla, (2011). “ DESIGN AND IMPLEMENTTION OF A SOFTWARE TOOL FOR SUSTAINABLE DEVELOPMENT ASSESSMENT “, Undergraduate Thesis, Department of Computer Science, University of Nicosia. 